\title{
Cerebellar Cognitive Affective Syndrome Improved by Donepezil
}

\author{
Yoichiro Nishida $^{1,2}$, Masaki Hizume ${ }^{1}$, Yuichi Fumimura ${ }^{1}$ and Tadashi Ichikawa $^{1}$
}

\begin{abstract}
:
Cerebellar damage can cause not only disturbance in motor control but also higher brain dysfunction known as cerebellar cognitive affective syndrome (CCAS). Although CCAS has a high prevalence, the precise mechanism and effective medications are unknown. We herein report a CCAS patient whose symptoms were ameliorated with the cholinesterase inhibitor donepezil. N-isopropyl-p- ${ }^{123} \mathrm{I}$-iodoamphetamine-singlephoton emission computed tomography showed improvement in hypoperfusion in the contralateral frontal and parieto-temporal lobes. Some projections with cholinergic transmission might form a functional connectivity between the cerebellum and contralateral association cortices, and cholinergic dysfunction is involved in CCAS pathophysiology. Donepezil might be worth considering for some CCAS patients.
\end{abstract}

Key words: cerebellar cognitive affective syndrome, cerebral blood flow, cholinesterase inhibitors, crossed hemispheric diaschisis, donepezil, SPECT

(Intern Med 58: 1003-1006, 2019)

(DOI: 10.2169/internalmedicine.1206-18)

\section{Introduction}

The cerebellum plays an important role in motor control. Cerebellar damage can cause disturbance in coordination, precision, and accurate timing of movements. In addition, anatomical, physiological, and functional neuroimaging studies have suggested that the cerebellum participates in the organization of higher-order functions. Some patients with cerebellar lesions experience memory impairment, attentional disturbance, executive dysfunction, and affective changes. These disorders of higher-order functions are recognized as cerebellar cognitive affective syndrome (CCAS) (1). The pathophysiology of CCAS and effective medications have been unclear. We experienced a patient with CCAS whose higher brain dysfunction was ameliorated using the cholinesterase inhibitor donepezil and whose brain functional imaging showed improvement in the contralateral association area.

\section{Case Report}

A 60-year-old cognitively-normal right-handed man with a 10-year history of hypertension suddenly experienced vertigo and vomiting. He was diagnosed with right cerebellar hemorrhaging with a rupture into the cerebroventricle (Supplementary material 1 ). He underwent craniotomy to remove the hematoma. His physical condition fully recovered, and his neurological deficits were minimal, with slight dysarthria and wide-based walking five months later. However, mild memory disturbance and severe higher brain dysfunction including deficits in mental, attentional, and executive functions remained even at 10 months after the craniotomy.

Although he was not evaluated by neuropsychological tests during the acute or subacute phase, these impairments, as his wife described, appeared acutely after the stroke, were relatively stable, and followed a non-progressive course for 12 months. Ten months after the surgery, his score on the Revised Hasegawa's Dementia Scale (HDS-R), consisting of 9 simple questions with a maximum score of 30, was 27 points, and his full-scale intelligence quotient by the Japa-

${ }^{1}$ Department of Neurology, Saitama Prefectural Rehabilitation Center, Japan and ${ }^{2}$ Department of Neurology and Neurological Science, Graduate School, Tokyo Medical and Dental University, Japan

Received: March 19, 2018; Accepted: July 16, 2018; Advance Publication by J-STAGE: December 18, 2018

Correspondence to Dr. Yoichiro Nishida, y-nishida.nuro@tmd.ac.jp 

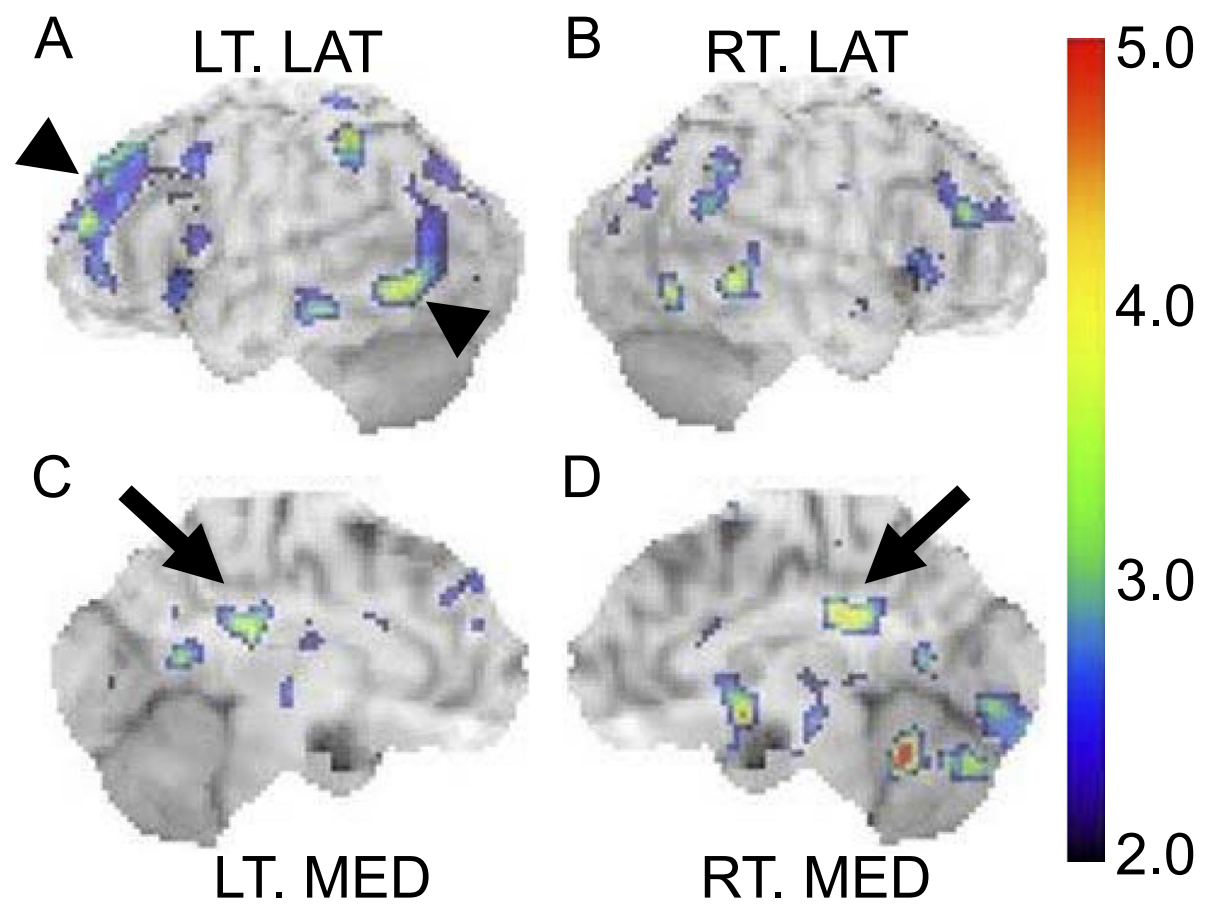

\begin{abstract}
Figure 1. The three-dimensional stereotactic surface projections analysis of N-isopropyl-p- ${ }^{123} \mathrm{I}$-iodoamphetamine-SPECT performed two months before taking donepezil. The lateral views (A, B) show reduced regional cerebral blood flow (rCBF) in the left superior frontal, left middle frontal, and left middle temporal gyri (arrow heads). The medial views $(C, D)$ reveal decreased $\mathrm{rCBF}$ in the bilateral posterior cingulate gyri (arrows). The right bar shows the severity of the decrease as the Z-score, calculated using the global mean as the reference value.
\end{abstract}

nese adult reading test was 100. However, he severely lacked initiative. He could accurately respond to some questions but needed some time to do so, and he never spoke first. His performance was also reduced on the backward condition of the digit span task; he was able to perform the task when three-digit numbers were given but could not when four-digit numbers were provided. His ability in the letter fluency task was also disturbed, as the number of words he was able to recall in 60 seconds was 4.3 on average. The percentage of correct answers and completion time were $60.5 \%$ and 160 seconds in the Visual Cancellation Task of a Letter, respectively. During the 4-disk Tower of Hanoi task, with a minimum number of steps of 15 , he completed the task in 359 seconds after 55 steps. His standard profile score on the Rivermead Behavioral Memory Test (RBMT) was 14 points. Although brain MRI showed no abnormality in the left frontal or parieto-temporal lobe (Supplementary material 2), N-isopropyl-p- ${ }^{123}$ I-iodoamphetamine-( $\left({ }^{123}\right.$ I-IMP-) single-photon emission computed tomography (SPECT) showed hypoperfusion not only in the right cerebellum but also in the left frontal and parieto-temporal lobes. A three-dimensional stereotactic surface projection (3 D-SSP) analysis revealed a reduced regional cerebral blood flow (rCBF) clearly in the left superior frontal, left middle frontal, and left middle temporal gyri (Fig. 1A) compared to that on the opposite side in the lateral views (Fig. 1B).

Based on these findings, he was diagnosed with CCAS, and the significant hypoperfusion in the left frontal and parieto-temporal lobes was recognized as crossed hemispheric diaschisis (2). In addition, the decreased $\mathrm{rCBF}$ in the bilateral posterior cingulate gyri in the medial views (Fig. 1C and D), along with his mild memory disturbance, suggested the presence of Alzheimer disease (AD) pathology. He started taking donepezil. Over the next four months, his higher brain dysfunction gradually ameliorated, and he clearly became self-motivated. He was able to talk about what he thought and wanted to do. He re-started golf training for recreation. His HDS-R improved to the full score of 30 points. He was able to perform the backward digit span task even with five-digit numbers. His word recall improved to 6.0 on average in the letter fluency task. His percentage of correct answers increased to $82.5 \%$ in the Visual Cancellation Task of a Letter, although the completion time was slightly prolonged to 189 seconds. His standard profile score on the RBMT slightly improved to 15 points.

We expected that donepezil would partially improve his AD symptoms accompanied by improvement of the reduced $\mathrm{rCBF}$ in the bilateral posterior cingulate gyri. However, follow-up using ${ }^{123}$ I-IMP-SPECT instead showed marked improvement in the hypoperfusion area and severity in the left frontal and parieto-temporal lobes (Fig. 2A), findings that did not markedly differ from those at the opposite side (Fig. 2B). Also surprising was the fact that the reduced $\mathrm{rCBF}$ in the bilateral posterior cingulate gyri was not improved (Fig. 2C and D); quite the contrary, the area and Zscores increased. 


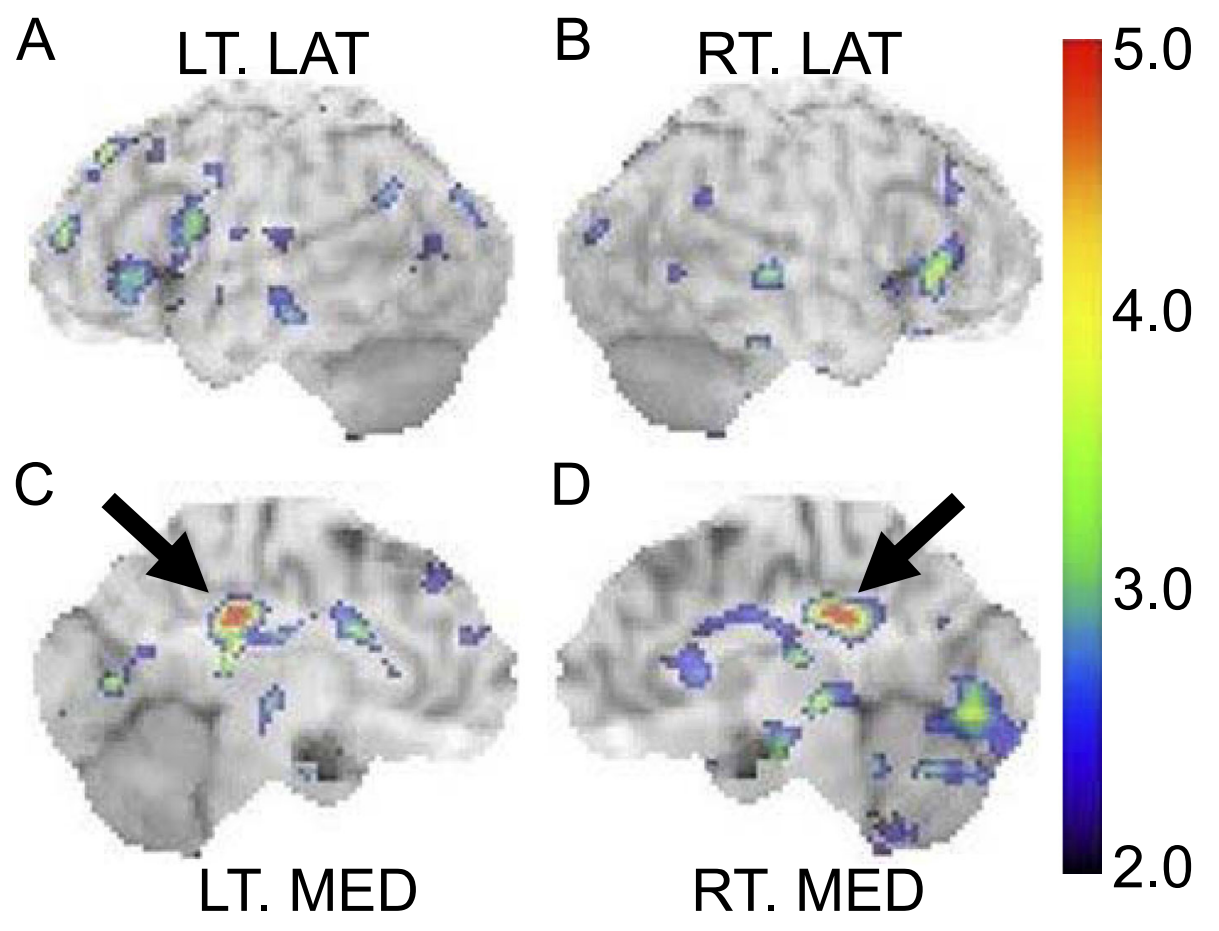

Figure 2. The three-dimensional stereotactic surface projections analysis performed four months after taking donepezil. The lateral views (A, B) show only tiny scattered spots with reduced regional cerebral blood flow (rCBF). The medial views $(C, D)$ show that the $\mathrm{rCBF}$ was lower than before the administration of donepezil in the bilateral posterior cingulate gyri (arrows). The right bar shows the severity of decrease as the $Z$-score, calculated using the global mean as the reference value.

We therefore attributed the amelioration of his higher brain dysfunction, specifically his executive function and working memory domains, rather than his recent and delayed recall function, to the improvement of neuronal dysfunction associated with CCAS and not AD pathology. Ten months after taking donepezil, improvements in his executive function and working memory were also observed in the 4-disk Tower of Hanoi task; this time, he completed the task in 20 steps after 137 seconds.

\section{Discussion}

Cerebellar regions have functional connectivity with various cerebral areas, including the contralateral association cortices (3-5). The constellation of deficits in CCAS suggests the disruption of neural circuits that link the prefrontal, posterior parietal, superior temporal, and limbic cortices with the cerebellum (1). In our patient, the areas where donepezil restored $\mathrm{rCBF}$ were mainly the left frontal and parieto-temporal association cortices. Although cholinergic deficits are well known to be present in AD and not CCAS, our patient showed marked improvements in his clinical symptoms and radiological findings with donepezil, suggesting that the cholinergic function is dysregulated in CCAS.

Although the prevalence of cognitive impairment caused by cerebellar hemorrhaging is very high $(83.3 \%)$ after an evaluation, even with a simple screening test like the MiniMental State Examination (6), no effective treatment has yet been identified. In the present patient, however, there seemed to be some improvements during the psychological tests. Improvements were also observed by the patient himself, his wife, and his doctors. Furthermore, ${ }^{123}$ I-IMP-SPECT showed that donepezil successfully ameliorated the reduced rCBF caused by CCAS and not AD pathology. We cannot completely deny the possibility that the improvement was due to the natural course of events after the surgery for cerebellar hemorrhaging and hydrocephalus. However, the probability of this being the case is low, as his improvement seemed to begin 12 months after his craniotomy, when donepezil was started. It is therefore possible that donepezil could thus be an effective treatment for his higher brain dysfunction.

In conclusion, some projections with cholinergic transmission may exist between the cerebellum and contralateral association cortices. Although further studies are required to confirm our findings, donepezil appears to be worth considering for the treatment of some CCAS patients.

The authors state that they have no Conflict of Interest (COI).

\section{References}

1. Schmahmann JD, Sherman JC. The cerebellar cognitive affective syndrome. Brain 121: 561-579, 1998.

2. Rousseaux M, Steinling M. Crossed hemispheric diaschisis in unilateral cerebellar lesions. Stroke 23: 511-514, 1992. 
3. Buckner RL, Krienen FM, Castellanos A, Diaz JC, Yeo BT. The organization of the human cerebellum estimated by intrinsic functional connectivity. J Neurophysiol 106: 2322-2345, 2011.

4. Schmahmann JD, Pandya DN. The cerebrocerebellar system. Int Rev Neurobiol 41: 31-60, 1997.

5. Ramnani N. The primate cortico-cerebellar system: anatomy and function. Nat Rev Neurosci 7: 511-522, 2006.

6. Maeshima S, Osawa A, Matsuda $\mathrm{H}$, et al. Cognitive dysfunction in patients with acute cerebellar hemorrhage. Dementia Japan 25: 164-169, 2011 (in Japanese, Abstract in English).

The Internal Medicine is an Open Access journal distributed under the Creative Commons Attribution-NonCommercial-NoDerivatives 4.0 International License. To view the details of this license, please visit (https://creativecommons.org/licenses/ by-nc-nd/4.0/).

(C) 2019 The Japanese Society of Internal Medicine Intern Med 58: 1003-1006, 2019 